\title{
CORRECTIONS TO 'CHARACTER THEORY OF FINITE GROUPS WITH TRIVIAL INTERSECTION SUBSETS (Vol 27, 515-524)
}

\author{
JOHN H. WALTER
}

1. The conditions (TI 1) and (TI 2) are stated for $\boldsymbol{H}=\mathfrak{R} G(\boldsymbol{D})$ and henceforth in the paper $\boldsymbol{H}$ is understood to be $\mathfrak{\Re}_{G}(\boldsymbol{D})$ when $\boldsymbol{D}$ is taken to be a T.I. subset of $\boldsymbol{G}$. Also in the definition of T. I. subset the condition is that $\boldsymbol{D} \cap \boldsymbol{D}^{\boldsymbol{G}} \neq \phi$ where $\phi$ is the empty set.

2. Just before formula (11), the symbol should read

$$
\left\{\varepsilon\left(\tau_{i}\right) \xi_{i} \mid \varepsilon\left(\tau_{i}\right) \xi_{i} \tau_{i}=\Xi\right\}
$$

3. In the statement of Proposition 8, the penultimate sentence should read: 'If $\boldsymbol{D}$ contains a section $\mathfrak{S}_{\boldsymbol{H}}(P)$ of a p-element $P$ belonging to a defect group $\boldsymbol{V}$ of $\mathfrak{B}^{G}$, then $\Im_{G}\left(\mathfrak{S}_{G},\left(\boldsymbol{D}^{G}, \mathfrak{B}^{G}\right)\right)$ contains all characters of zero height in $\mathfrak{B}^{G} .{ }^{\prime}$ It is required to know that $\Xi(R) \not \equiv$ in the proof for a character $\Xi$ of $\mathfrak{B}^{G}$ for an appropriate $p$ regular element $R$ in order to have $\Xi(P R) \neq 0$ where $P R \in D$. The assumption of zero height is need to justify this step.

The author is grateful to Professor J.G. Thompson and Warren Wong for their comments. There is a substantial overlap with the article and Wong's 'Exceptional Character Theory and the Theory of Blocks', Math. Zeit., 91, 363-379 (1966).

Received December 10, 1966. 